\title{
BMJ Open Evolution of pain at 3 months by oral resveratrol in knee osteoarthritis (ARTHROL): protocol for a multicentre randomised double-blind placebo- controlled trial
}

Christelle Nguyen, ${ }^{1,2,3}$ Isabelle Boutron, ${ }^{1,4,5}$ Gabriel Baron, ${ }^{1,5}$ Emmanuel Coudeyre, ${ }^{6}$ Francis Berenbaum, ${ }^{7,8,9}$ Serge Poiraudeau, ${ }^{1,2,10,11}$ François Rannou ${ }^{1,2,3}$

To cite: Nguyen C, Boutron I, Baron G, et al. Evolution of pain at 3 months by oral resveratrol in knee osteoarthritis (ARTHROL): protocol for a multicentre randomised double-blind placebocontrolled trial. BMJ Open 2017;7:e017652. doi:10.1136/ bmjopen-2017-017652

- Prepublication history and additional material for this paper are available online. To view these files please visit the journal (http://dx.doi.org/10. 1136/bmjopen-2017-017652)

SP deceased.

Received 5 May 2017

Revised 19 July 2017

Accepted 2 August 2017

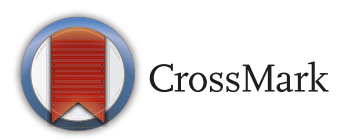

For numbered affiliations see end of article.

Correspondence to Dr Christelle Nguyen; christelle.nguyen2@aphp.fr

\section{ABSTRACT}

Introduction 0steoarthritis $(\mathrm{OA})$ pathophysiology is driven in part by joint inflammation. Resveratrol has in vitro antiinflammatory properties. We aim to assess the efficacy of oral resveratrol for knee pain at 3 months in people with knee OA.

Methods and analysis We will conduct a randomised double-blind placebo-controlled trial. Overall, 164 individuals with knee OA fulfilling 1986 American College of Rheumatology criteria will be recruited in three tertiary care centres in France and randomised to receive oral resveratrol, $40 \mathrm{mg}$ (two caplets) two times per day for 1 week, then $20 \mathrm{mg}$ (one caplet) two times per day or a matching placebo for a total of 6 months. Randomisation will be centralised and stratified by centre. The allocation ratio of assignments will be $1: 1$. The primary outcome will be the mean change from baseline in knee pain on a self-administered 11-point pain Numeric Rating Scale at 3 months. Secondary outcomes will be the mean change in knee pain at 6 months, the function subscore of the Western Ontario and McMaster Universities Arthritis Index score, patient global assessment, proportion of responders according to the 0steoarthritis Research Society International-Outcome Measures in Rheumatology criteria at 3 and 6 months, and self-reported number of intra-articular injections of corticosteroids or hyaluronic acid and consumption of analgesics and non-steroidal anti-inflammatory drugs since the last contact. Other interventions will be allowed and self-reported. Adherence will be monitored by capsule counts and a booklet and adverse events recorded at 3 and 6 months. Statisticians, treating physicians and participants will be blinded to the allocated treatment.

Ethics and dissemination The oral resveratrol in knee osteoarthritis (ARTHROL) trial has been authorised by the AgenceNationale de Sécurité du Médicament et des Produits de Santé and ethics were approved by the Comité deProtection des Personnes Île-de-France III. The findings of the study will be published in a peer-reviewed journal and disseminated at conferences. The design of ARTHROL will warrant the translation of its findings into clinical practice.

Trial registration number ClinicalTrials.gov identifier: NCT02905799. Pre-results. First received: 14 September
Strengths and limitations of this study

- First randomised controlled trial to assess the effects of oral resveratrol on pain in knee osteoarthritis.

- A design to facilitate the translation of findings into clinical practice.

- Innovative new formulation of oral resveratrol to improve its bioavailability.

- Selection of primary and secondary efficacy outcomes in accordance with Outcome Measures in Rheumatology recommendations and Core Outcome Measures in Effectiveness Trials initiative for phase III clinical trials in knee osteoarthritis.

- Participants will be recruited from tertiary care centres and may not be fully representative of the population with knee osteoarthritis in France.

2016. Last updated: 16 September 2016. Status: not yet recruiting.

\section{INTRODUCTION}

In the 2015 Global Burden of Disease Study, musculoskeletal disorders were identified among the five main contributors to disability-adjusted life years. ${ }^{1}$ Knee osteoarthritis (OA) is one of the most disabling joint disorders in Western countries ${ }^{2}$ and OA is the first cause of disability in people over 40 years old in France. ${ }^{3}$

OA pathophysiology is in part driven by local joint inflammation leading to severe tissue damage. No efficient treatment exists for structural changes in OA; the only treatments are for painful symptoms and are mainly acetaminophen, non-steroidal anti-inflammatory drugs (NSAIDs) and weak opioids. Unfortunately, acetaminophen is weakly effective, with a poor effect size of 0.10 , and recent data highlighted its potential cardiovascular 
adverse effects. ${ }^{45}$ For NSAIDs, serious cardiovascular and digestive side effects do not support their prescription for long duration. An optimised treatment for OA should be efficient for both pain and inflammation, with minimal adverse effects.

Resveratrol is a molecule of interest because it has in vitro and in vivo anti-inflammatory and chondroprotective properties. ${ }^{67}$ Resveratrol is available over the counter in France as a dietary supplement. No serious toxicity has been reported. In the field of rheumatic diseases, in vitro evidence supports anti-inflammatory, anticatabolic, antiapoptotic and antioxidative properties of resveratrol in various articular cell types, along with immunomodulation properties for T and B lymphocytes. ${ }^{8-20}$ Consistently, resveratrol administered intra-articularly has shown chondroprotective effects in preclinical models of OA, mediated by decreased production of proinflammatory and prodegradative soluble factors, and modulation of cellular and humoral responses. ${ }^{21-23}$ In clinical research, resveratrol has been evaluated in ageing, cancer, neurodegenerative diseases, menopausal conditions, and cardiovascular and liver diseases. ${ }^{6}$ The doses used in these trials were variable and not adjusted to the low bioavailability of oral formulations.

New formulations of resveratrol have allowed for an increase in oral resveratrol bioavailability. ${ }^{24}$ The plasmatic peak is 10-fold increased and blood concentration remains at significant levels for several hours. We hypothesised that oral resveratrol in a new formulation could reduce knee pain at 3 months as compared with placebo in people with knee OA.

\section{METHODS AND ANALYSIS Design overview}

This is a prospective, parallel-group, double-blind, randomised controlled multicentre study. Duration of follow-up for each participant will be 6 months postrandomisation. The study will be reported according to the Consolidated Standards of Reporting Trials statement. ${ }^{25}$

\section{Setting and participants}

Participants will be prospectively recruited among inpatients and outpatients from Rheumatology and Rehabilitation departments of three tertiary care centres in France with expertise in OA management (Cochin and Saint-Antoine Hospitals, Paris, France and Gabriel-Montpied Hospital, Clermont-Ferrand, France), by advertising on the internet and in the media (newspapers and health magazines) and by using posters in each investigating centre. People interested in participating in the study will be invited to contact a biomedical research technician by phone or email. In addition, the computerised medical records of each investigating centre will be searched from 2015 to 2017, and patients with the key words 'knee OA' in the records will be invited to participate in the study by phone or mail by the biomedical research technician. The number of patients treated yearly for knee OA in the participating centres is approximately 2000. The biomedical research technician will check for eligibility criteria, then, if appropriate, set up a face-to-face baseline visit with one of the investigators, a senior specialist in rehabilitation and/or rheumatology. The main eligibility criteria will be knee OA fulfilling 1986 American College of Rheumatology criteria, pain on a self-administered 11 -point pain Numeric Rating Scale (NRS) $\geq 40 / 100$, symptom duration $\geq 1$ month and Kellgren and Lawrence X-ray score 1, 2 or 3 . A complete description of the inclusion and non-inclusion criteria is presented in online supplementary appendix 1 . Patients excluded for temporary reasons can be rescreened.

\section{Experimental group}

$40 \mathrm{mg}$ (two caplets) of resveratrol will be administered orally two times per day, $30 \mathrm{~min}$ before a meal with a glass of water, for 1 week, then $20 \mathrm{mg}$ (one caplet) two times per day for a total of 6 months. Pharmacokinetics, bioavailability and toxicity of trans-resveratrol formulation used in the oral resveratrol in knee osteoarthritis (ARTHROL) trial have been previously described in a phase I clinical trial. ${ }^{24}$ Briefly, 15 healthy volunteers received a single dose of $40 \mathrm{mg}$ of oral trans-resveratrol in two forms (soluble galenic formulation or dry powder). The single dose of the soluble trans-resveratrol was well absorbed and elicited biologically efficient blood levels $(0.1-6 \mu \mathrm{M})$ for several hours. The soluble formulation led to 8.8-fold higher trans-resveratrol levels in plasma versus the powder. We have made substantial modifications to the administration scheme as compared with the one tested in the phase I clinical trial: (1) because trans-resveratrol is metabolised into glucuronide and sulfate conjugates coupled to renal elimination, we hypothesised that giving a loading dose for 1 week may allow attaining the drug effect more rapidly, and (2) for the maintenance dose, we chose $40 \mathrm{mg}$ a day as tested in the phase I clinical trial, but in two doses, because the half-life of the soluble galenic formulation of trans-resveratrol is $79 \mathrm{~min}$ only. Resveratrol will be freely supplied by the Yvery Laboratory (patent number WO 2012/007252, Marseille, France). Resveratrol is considered a dietary supplement and is available over the counter. No marked toxicity has been reported. ${ }^{26}$ The caplets used in this study will be exactly the same as those already available on the French market. They will be stored in their original packaging at room temperature, protected from humidity, light and excessive heat. A box containing 7 pillboxes of 60 caplets each of resveratrol will be provided to each person randomised to the experimental group. Individuals will be asked to return the pillboxes for capsule counts at the 3-month and 6-month visits and to self-report adherence by completing a booklet. However, no specific measures will be taken to enhance adherence.

\section{Control group}

The Yvery Laboratory will supply the placebo and ensure that it has a similar condition and taste as resveratrol. Two 
caplets of placebo will be administered orally two times per day for 1 week, then one capsule two times per day for a total of 6 months. A box containing 7 pillboxes of 60 caplets each of placebo will be provided to each person randomised to the control group and stored under the same conditions as resveratrol.

\section{Co-interventions}

Pharmacological and non-pharmacological treatments usually prescribed for knee OA will be authorised. Rescue medications (analgesics and NSAIDs), joint injections (hyaluronic acid and corticosteroids), symptomatic slow acting drugs for OA (SYSADOA) and non-pharmacological co-interventions including brace, insoles, walking aids, physiotherapy, home-based therapeutic exercises and weight loss will be assessed by using a standardised checklist and recorded in the electronic case report form (eCRF).

\section{Outcomes}

Primary and secondary efficacy outcomes have been selected in accordance with Outcome Measures in Rheumatology (OMERACT) ${ }^{27}$ and Osteoarthritis Research Society International (OARSI) recommendations ${ }^{28}$ and the Core Outcome Measures in Effectiveness Trials initiative for phase III clinical trials of knee OA. As recommended, the outcomes include those for pain, physical function and patient global assessment. The primary efficacy outcome is the mean change from baseline in mean knee pain in the previous 48 hours on a self-administered 11-point pain NRS (0, no pain, to 100 , maximal pain) at 3 months. The secondary efficacy outcomes are the mean change in mean knee pain on a pain NRS at 6 months, the mean change in the function subscore of the self-administered Western Ontario and McMaster Universities Arthritis Index (WOMAC) questionnaire at 3 and 6 months (the French version of the questionnaire), ${ }^{29}$ the mean change in patient global assessment at 3 and 6 months on a self-administered 11-point global assessment NRS (0, worst possible, to 100 , best possible), the proportion of responders according to the OARSI-OMERACT at 3 and 6 months, ${ }^{30}$ the self-reported number of intra-articular injections of corticosteroids or hyaluronic acid and the self-reported consumption of analgesics (non-opioid, weak and strong opioids) and NSAIDs since the last contact on a self-administered four-class scale (never, several times a month, several times a week, daily) at 3 and 6 months. Information about the WOMAC questionnaire and OARSI-OMERACT response is presented in online supplementary appendix 2. For participants who discontinue or deviate from intervention protocols, the same outcome data will be collected if possible.

\section{Randomisation and allocation concealment}

Individuals who meet the inclusion criteria and agree to participate will be randomly assigned to the resveratrol or placebo group at the inclusion visit. The allocation ratio of assignments will be 1:1. Participants, care providers, data collectors, outcome assessors and statisticians will be blinded to the allocated group. The randomisation sequence will be computer generated by a statistician of the Centre d'Épidémiologie Clinique. The list will be stratified by centres with variable block sizes. The randomisation process will be centralised at the coordinating office (Unité de Recherche Clinique, Cochin Hospital), which will have no involvement in the enrolment, follow-up or assessment of participants. Only the independent statistician of the Centre d'Épidémiologie Clinique, the computer programmer at the coordinating office who will implement the sequence assignment in the secure eCRF, and the Yvery Company will have access to the randomisation list. The Yvery Company will label the resveratrol and placebo caplets and provide them with strictly identical presentations to each centre for the whole research duration. In each centre, the investigator will blindly deliver the medication to patients enrolled according to their randomisation number, at once, for the whole research duration. The sequence will be concealed by use of a computer interface implemented in the eCRF. Treatment administration and clinical monitoring of the experimental products will be the same in the experimental and control groups.

Blinding can be broken only if the investigator deems it necessary for the safe management of a specific medical condition of a subject, and whenever possible, the methodologist and sponsor will be consulted before breaking the blind. If the blind is broken for any reason during the study, the moment at which the blind was broken and all other relevant information will be documented by the investigative site and other sponsor designees, as appropriate. The reason for breaking the blind will be indicated and justified in the source documentation and in the eCRF.

\section{Statistical aspects}

The sample size is estimated at 164 patients. We have predicted a difference in mean change from baseline of 15 points on the pain NRS between resveratrol and placebo groups, with a SD of 27 points, and a power of $90 \%$, corresponding to 69 patients in each arm. Considering a $15 \%$ lost to follow-up, we will need to enrol an estimated 82 patients for each arm. Fifteen points on pain NRS is considered the minimal clinically perceived difference in pain for patients with knee OA. All analyses will be performed on an intent-to-treat basis, in that all patients will be considered in the analysis and will be analysed in the group to which they had been assigned. For descriptive analyses, qualitative variables will be reported with absolute and relative frequencies and quantitative variables with median (IQR). To compare differences in changes in values between the two groups for quantitative variables, a constrained longitudinal data analysis will be used. In this model, both the baseline and postbaseline values will be modelled as dependent variables (the constrained longitudinal data analysis model assumes that both the baseline and postbaseline measurements 
are jointly multivariate normally distributed because the baseline value is treated as part of the response vector). The true baseline means will be constrained to be the same for the two treatment groups. This analysis provides an adjustment for the observed baseline difference in estimating the treatment effects. Random effects at patient and centre levels will be added to these models. Results will be expressed as differences in mean change from baseline with 95\% CI at 3 and 6 months. The constrained longitudinal data analysis model can include all randomised subjects with a baseline or postbaseline value. Such methods based on maximum likelihood are consistent under the missing-at-random assumption. Qualitative outcomes will be analysed by a mixed logistic regression model with a random effect at centre levels. Data analysis will involve use of SAS V.9.4. Blinded statisticians will perform the statistical analyses at an independent centre (Centre d'Épidémiologie Clinique, Paris
Descartes, Hôpital Hôtel-Dieu). The statistical analysis will be further detailed in a dedicated Statistical Analysis Plan before any analysis is undertaken.

\section{Participant timeline}

Schedule of enrolment, interventions and assessments is shown in Table 1 .

Baseline visit

Inclusion and non-inclusion criteria will be validated at baseline by the investigator during a face-to-face visit. The individual will be informed and the written consent collected by the investigator. Then, the participant will be enrolled and randomised. Specific additional clinical examination, laboratory tests or imaging will not be required for the purpose of the study. Information regarding demographics (age, gender, body mass index, education and employment status), medical history (date

Table 1 Schedule of enrolment, interventions and assessments

\begin{tabular}{|c|c|c|c|}
\hline & \multicolumn{3}{|c|}{ Study period } \\
\hline & Enrolment & Postallocation & Close-out \\
\hline Timepoints & Month 0 & Month 3 & Month 6 \\
\hline Informed consent & $X$ & - & - \\
\hline Allocation & $\mathrm{X}$ & - & - \\
\hline Delivery of a participating card & $\mathrm{X}$ & - & - \\
\hline Instructions to keep and return the pillboxes & $\mathrm{X}$ & - & - \\
\hline \multicolumn{4}{|l|}{ Interventions } \\
\hline \multicolumn{4}{|l|}{ Oral resveratrol } \\
\hline Oral placebo & & & \\
\hline
\end{tabular}

\section{Assessments}

Baseline variables

Demographics

Medical history

Outcome measures (analyses planned)

$\begin{array}{llll}\text { Knee pain } & X & X & X \\ \text { WOMAC function subscore } & X & X & X \\ \text { Patient global assessment } & X & X & X \\ \text { OARSI-OMERACT response } & - & X & X \\ \text { Analgesics and NSAIDs consumption } & X & X \\ \text { Injections of hyaluronic acid and/or corticosteroids } & X & X \\ \text { Collected variables (no analyses planned) } & & X \\ \text { Symptomatic slow acting drugs for OA consumption } & & X \\ \text { Non-pharmacological co-interventions } & X & X \\ \text { Adverse events } & - & X \\ \text { Adherence } & - & X\end{array}$

NSAIDs, non-steroidal anti-inflammatory drugs; OA, osteoarthritis; OARSI, Osteoarthritis Research Society International; OMERACT, Outcome Measures in Rheumatology; WOMAC, Western Ontario and McMaster Universities Arthritis Index. 
of diagnosis, symptoms duration, side affected, medical history, surgery and trauma history of the affected knee, X-ray findings including Kellgren and Lawrence grade and OA location (ie, femorotibial medial, femorotibial lateral and/or patellofemoral $\mathrm{OA}$ )), medications in the previous 3 months (analgesics, NSAIDs and intra-articular injections of hyaluronic acid and/or corticosteroids and SYSADOA) and current non-pharmacological co-interventions (brace, insoles, walking aids, physiotherapy, home-based therapeutic exercises and weight loss) will be recorded in the eCRF by using a standardised checklist. Baseline values for prespecified assessment criteria will be collected by using printed self-administered questionnaires and data will be entered in the eCRF by a biomedical research technician. The investigator will deliver the experimental product to the participants according to their randomisation number and give them a participating card in a clinical trial. Participants will be asked to keep and return the pillboxes for capsule counts at the 3-month and 6-month visits and to self-report adherence by completing a booklet.

\section{Three-month and 6 month visits}

The investigator will assess participants during a face-toface visit. Values for prespecified assessment criteria will be collected at 3 and 6 months by using printed self-administered questionnaires, and data will be recorded in the eCRF by a biomedical research technician. In addition, the investigator will record adverse events (AEs) since the last contact by asking an open-ended question ("Did you have any adverse events since the last contact?"), count the caplets remaining in the pillboxes, check the self-reported adherence booklet and assess non-pharmacological co-interventions by using a standardised checklist. In the event that the participant missed the appointment, self-administered questionnaires, AEs, capsule counts and non-pharmacological co-interventions will be collected by mail, email or phone by a biomedical research technician and recorded. To reduce the amount of missing data, promote participant retention and complete the follow-up, reminder newsletters will be sent once a month by mail or email to inform participants of the progression of the study.

\section{End of the research}

At the end of the research, patients will be advised to continue their usual medical follow-up with their treating physician. Ending a subject's participation will not affect the normal medical management in any way. No exclusion period for another biomedical research will be required. At the end of the study, participants will be informed of the results on request.

\section{Data management}

\section{Data collection}

Data will be entered into an eCRF, completed by the investigator during each visit. Data from printed self-administered questionnaires will be entered in the eCRF by a biomedical research assistant after the visits. The investigator must give an explanation for each missing data. Changes in the data in the eCRF will be tracked. Discordant data in the eCRF will be corrected by queries.

\section{Data monitoring}

In accordance with the French Good Clinical Practices, the sponsor, DRCD, is responsible for obtaining the permission of all parties involved in the research to guarantee direct access to all locations where the research is carried out, the source data, the source documents and the reports, with the goal of quality control and audit by the sponsor. In accordance with the legislative and regulatory provisions in force (Articles L.1121-3 and R.5121-13 of the French Public Health Code), the investigators will make available to those in charge of monitoring, quality control and audit relating to the biomedical research the documents and personal data strictly necessary for these controls. Source documents are defined as any original document or object that can prove the existence or accuracy of a piece of information or a fact recorded during the research.

\section{Quality control}

A clinical research associate appointed by the sponsor will be responsible for the proper conduct of the research and for collecting and documenting, recording and reporting the data generated in writing, in accordance with the standard operating procedures applied within the DRCD and in accordance with French Good Clinical Practices as well as with the legislative and regulatory provisions in force. The investigator and the members of the investigator's team agree to make themselves available during quality control visits carried out at regular intervals by the clinical research associate. During these visits, the following elements will be reviewed: written consent, compliance with the research protocol and with the procedures defined therein, quality of the data collected in the eCRF including accuracy, missing data, consistency of the data with the source documents (medical files, appointment books, original copies of laboratory results, among others) and management of the treatments used.

\section{ETHICS AND DISSEMINATION Ethical considerations}

Methods for obtaining information and consent from research participants

In accordance with Article L.1122-1-1 of the French Public Health Code, no biomedical research can be carried out on a person without free and informed consent obtained in writing after the person has been given the information specified in Article L.1122-1 of said code. The investigator or a doctor representing the investigator obtains the free and informed consent, in writing, of the individual before their inclusion in the research. The information sheet and a copy of the consent form signed and dated by the research participant and by the investigator or the doctor 
representing the investigator are given to the individual before their participation in the research. In addition, the investigator will specify in the research participant's medical file the methods used for obtaining consent as well as the methods used for providing information with the goal of obtaining consent. The investigator will retain the original signed and dated copy of the participant's consent form.

\section{Data confidentiality}

Those responsible for biomedical research quality control (Article L.1121-3 of the French Public Health Code) will take all necessary precautions to ensure the confidentiality of information about the experimental medications, the research, the research subjects and in particular the identity of the participants and the results obtained. Investigators are subject to professional secrecy (in accordance with the conditions set out in Articles 226-13 and 226-14 of the Penal Code). During or after the biomedical research, the data collected for the research participants and sent to the sponsor by the investigators (or any other specialised parties) will be made non-identifying. Anonymisation of the patients will be ensured by using a code number and initials, reported on each needed document for the research, or by erasing nominative data on copies of source documents. Under no circumstances should the names and addresses of the subjects involved be shown. The sponsor will ensure that each research participant has given permission in writing for access to their personal information that is strictly necessary for the quality control of the research. Access to the eCRF will be restricted by an access code and a personal and unique password system for each user. Each investigator will, in addition, have access to a specific profile that attributes or withholds access to certain functions of the system (entering data, or simply viewing the data of the enrolled participant or all the study data, possibility of change and validation by the clinical research associate, etc). Data will be stored on a secure server, with data encrypted during transmission and automatic internal saving of a copy on the server that will host the eCRF. This research falls under the Méthodologie de référence according to the provisions of Article 54, paragraph 5 of modified Law no 78-17 of 6 January 1978 relating to information technology, data files and privacy. This change was approved in a decision on 5 January 2006. AP-HP, the research sponsor, has signed a commitment to comply with the Méthodologie de référence. Specific documents for biomedical research will be archived by the investigator and the sponsor for 15 years after the end of the research.

\section{Legal obligations}

AP-HP is the sponsor of this research and by delegation, the DRCD performs the research's missions in accordance with Article L.1121-1 of the French Public Health Code. For this biomedical research relating to a medication for human use and prior to starting the research, AP-HP has obtained the favourable opinion of the Comité de Protection des Personnes (CPP) Ille-de-France III, within the scope of its authority and in accordance with the legislative and regulatory provisions in force. AP-HP has also obtained authorisation from the Agence Nationale de Sécurité du Médicament et des produits de santé (ANSM) (French Health Products Safety Agency); registration number RCB 2016-A01310-51). AP-HP has signed a commitment to comply with the Méthodologie de reference. AP-HP will make a standard declaration to the Commission Nationale de l'Informatique et des Libertés.

\section{Modifications to the research}

Any substantial modification to the protocol by the coordinating investigator must be sent to the sponsor for approval. After approval is given, the sponsor must obtain, before starting the research, a favourable opinion from the CPP and authorisation from the ANSM within the scope of their respective authorities. The information sheet and the consent form can be revised if necessary, particularly with substantial modification to the research or if adverse reactions occur.

\section{Safety considerations}

The investigator will record all serious AEs (SAEs) and non-SAEs since the last contact by asking an open-ended question ("Did you have any adverse events since last contact?") during face-to-face visits at 3 and 6 months. In the event that the participant missed the appointment, AEs will be requested by mail, email or phone by a biomedical research technician and recorded. All SAEs and non-SAEs will be recorded in the 'Adverse events' section of the eCRF by the investigator.

\section{Notification of an SAE}

The investigator will notify the sponsor, immediately on the day when he/she becomes aware, of any SAE, except those that are prespecified (see below). The investigator must report all SAEs that occur in research participants on the date of the first administration of an investigational product and throughout the period when the participant is monitored. SAEs that do not require immediate notification to the sponsor are recorded in the 'Adverse events' section of the eCRF. They include events associated with (1) the normal and natural evolution of the pathology including scheduled medical visits for the follow-up of knee OA, scheduled hospitalisations for the routine treatment of knee OA (joint injection and rehabilitation), and not related to a worsening of the condition, and expected symptoms secondary to knee OA worsening such as joint pain, joint effusion, OA flare, walking difficulties or surgical knee joint replacement for $\mathrm{OA}$; (2) special circumstances including hospitalisations for pre-existing conditions, surgery scheduled prior to the research, social or administrative purposes or admission to the emergency room less than 12 hours and (3) AEs likely to be associated with the treatments prescribed as part of the patient's care during the monitoring of the research, including AEs related to rescue medications 
(analgesics and NSAIDs) or joint injections (hyaluronic and corticosteroids) that include increased pain, joint swelling, mild joint effusion that can last a few days, skin flush following corticosteroid injections that can last a few hours and exceptionally, septic arthritis or allergic reaction.

\section{Investigation of an SAE}

The investigator will document the SAE as thoroughly as possible and provide the medical diagnosis by using a specific SAE form. The investigator will assess the severity of the SAE: (1) mild, tolerated by the participant, does not interfere with daily activities; (2) moderate, sufficiently uncomfortable to affect daily activities and (3) serious, preventing daily activities. The investigator will assess the causality relation between the SAE and the clinical trial. The method the investigator uses will be based on WHOUppsala Monitoring Centre method and will include the following four causality terms: (1) certain, (2) probable/ likely, (3) possible and (4) unlikely (not excluded). Their comprehensive definition is provided in online supplementary appendix 3. The sponsor represented by the Vigilance department will continuously assess the safety of the clinical trial throughout the trial. The sponsor is responsible for assessing (1) the seriousness of all AEs reported and (2) the causality relation between the SAE and the acts/procedures/tests added by the clinical trial.

\section{Investigation of an AE}

All SAEs considered by the investigator and/or the sponsor to be possibly related to the act/procedures/ tests/products administered, specific to the clinical trial, can be reasonably considered suspected adverse reactions (SARs). Any SAR whose nature, severity or outcome is not consistent with the information related to the acts/procedures/and or products administered during the clinical study is considered unexpected. The sponsor represented by the Vigilance department will assess the expected/ unexpected nature of an SAR according to the information described in online supplementary appendix $4 .{ }^{26}$ The sponsor reports any suspected unexpected serious adverse reaction, within the legal deadline, to the ANSM and CPP.

\section{Dissemination plan}

We aim to publish the results of ARTHROL trial in a peer-reviewed journal and present the findings to physicians who manage knee $\mathrm{OA}$ at national and international conferences. The investigators will be involved in drafting manuscripts, abstracts, press releases and any other publications arising from the trial. Authorship will be determined in accordance with the International Committee of Medical Journal Editors guidelines. There will be no intended use of professional writers. AP-HP is the owner of the data, which cannot be used or disclosed to a third party without prior approval from the AP-HP. The full original protocol in English and the full dataset will be available by contacting the coordinating investigator, Prof
François Rannou (francois.rannou@aphp.fr). Statistical codes will be available by contacting the biostatistician of the study, Dr Gabriel Baron (gabriel.baron@aphp.fr).

The ARTHROL study will be the first to assess the clinical effects of oral resveratrol in knee OA. If the results are positive, resveratrol will represent an interesting and safe alternative for treating painful knee OA.

\section{Author affiliations}

${ }^{1}$ Faculté de Médecine, Sorbonne Paris Cité, Université Paris Descartes, Paris, France ${ }^{2}$ Service de Rééducation et de Réadaptation de l'Appareil Locomoteur et des Pathologies du Rachis, AP-HP, Hôpitaux Universitaires Paris Centre-Groupe Hospitalier Cochin, Paris, France

${ }^{3}$ INSERM UMR 1124, Laboratoire de Pharmacologie, Toxicologie et Signalisation Cellulaire, Faculté des Sciences Fondamentales et Biomédicales, Centre Universitaire des Saints-Pères, Paris, France

${ }^{4}$ AP-HP, Hôpital Hôtel-Dieu, Centre d'Épidémiologie Clinique, Paris, France ${ }^{5}$ INSERM UMR 1153, Centre de Recherche Épidémiologie et Statistique, Sorbonne Paris Cité, METHODS Team, Paris, France

${ }^{6}$ Service de Médecine Physique et de Réadaptation, Centre Hospitalo-Universitaire de Clermont-Ferrand, INRA, Université Clermont Auvergne, Clermont-Ferrand,

France

${ }^{7}$ Université Paris Pierre et Marie-Curie, Sorbonne Paris Cité, Paris, France

${ }^{8}$ Service de Rhumatologie, AP-HP, Hôpital Saint-Antoine, Paris, France

${ }^{9}$ INSERM UMR 938, DHU i2B, Paris, France

${ }^{10}$ INSERM UMR 1153, Centre de Recherche Épidémiologie et Statistique, Sorbonne

Paris Cité, ECaMO Team, Paris, France

${ }^{11}$ Institut Fédératif de Recherche sur le Handicap, Paris, France

Acknowledgements The authors thank Alexandra Bruneau from URC-CIC Paris Descartes Necker/Cochin for implementation, monitoring and data management and Laura Smales for professional copyediting.

Contributors Conception and design of the study: $\mathrm{CN}, \mathrm{IB}, \mathrm{GB}, \mathrm{EC}, \mathrm{FB}, \mathrm{SP}$ and FR. Drafting of the original protocol: $\mathrm{CN}, \mathrm{IB}, \mathrm{GB}, \mathrm{SP}$ and FR. Coordination of the study: $\mathrm{CN}$ and FR. Design of the statistical analysis plan: IB and GB. Drafting of the present manuscript: CN and IB. Final approval: CN, IB, GB, EC, FB and FR.

Funding This work was supported by the French Ministry of Health (Programme Hospitalier de Recherche Clinique 2015, project no 15-15-0234) and sponsored by the Département de la Recherche Clinique et du Développement (DRCD) of the AssistancePublique-Hôpitaux de Paris (AP-HP). The Yvery Laboratory will supply the resveratrol and the placebo. The funding source and the Yvery Laboratory will not be involved in the study design; collection, management, analysis and interpretation of data; or writing of the report; nor decision to publish the results.

Competing interests None declared.

Ethics approval Comité de Protection des Personnes Île-de-France III.

Provenance and peer review Not commissioned; externally peer reviewed.

Open Access This is an Open Access article distributed in accordance with the Creative Commons Attribution Non Commercial (CC BY-NC 4.0) license, which permits others to distribute, remix, adapt, build upon this work non-commercially, and license their derivative works on different terms, provided the original work is properly cited and the use is non-commercial. See: http://creativecommons.org/ licenses/by-nc/4.0/

(C) Article author(s) (or their employer(s) unless otherwise stated in the text of the article) 2017. All rights reserved. No commercial use is permitted unless otherwise expressly granted.

\section{REFERENCES}

1. Disease GBD, Injury I, Global PC.GBD 2015 Disease and Injury Incidence and Prevalence Collaborators. Global, regional, and national incidence, prevalence, and years lived with disability for 310 diseases and injuries, 1990-2015: a systematic analysis for the Global Burden of Disease Study 2015. Lancet 2016;388:1545-602.

2. Bijlsma JW, Berenbaum F, Lafeber FP. Osteoarthritis: an update with relevance for clinical practice. Lancet 2011;377:2115-26. 
3. Palazzo C, Ravaud JF, Papelard A, et al. The burden of musculoskeletal conditions. PLoS One 2014;9:e90633.

4. Richette P, Latourte A, Frazier A. Safety and efficacy of paracetamol and NSAIDs in osteoarthritis: which drug to recommend? Expert Opin Drug Saf 2015;14:1259-68.

5. Roberts E, Delgado Nunes V, Buckner S, et al. Paracetamol: not as safe as we thought? A systematic literature review of observational studies. Ann Rheum Dis 2016;75.

6. Vang O, Ahmad N, Baile CA, et al. What is new for an old molecule? Systematic review and recommendations on the use of resveratrol. PLOS One 2011;6:e19881.

7. Nguyen C, Savouret JF, Widerak M, et al. Resveratrol, potential therapeutic interest in joint disorders: a critical narrative Review. Nutrients 2017;9:45.

8. Dave M, Attur M, Palmer G, et al. The antioxidant resveratrol protects against chondrocyte apoptosis via effects on mitochondrial polarization and ATP production. Arthritis Rheum 2008;58:2786-97.

9. Li X, Phillips FM, An HS, et al. The action of resveratrol, a phytoestrogen found in grapes, on the intervertebral disc. Spine 2008;33:2586-95.

10. Malemud CJ. Inhibitors of stress-activated protein/mitogen-activated protein kinase pathways. Curr Opin Pharmacol 2007;7:339-43.

11. Mengshol JA, Vincenti MP, Coon $\mathrm{Cl}$, et al. Interleukin-1 induction of collagenase 3 (matrix metalloproteinase 13) gene expression in chondrocytes requires p38, c-Jun $\mathrm{N}$-terminal kinase, and nuclear factor kappaB: differential regulation of collagenase 1 and collagenase 3. Arthritis Rheum 2000;43:801-11.

12. Shakibaei M, Mobasheri A, Buhrmann C. Curcumin synergizes with resveratrol to stimulate the MAPK signaling pathway in human articular chondrocytes in vitro. Genes Nutr 2011;6:171-9.

13. Csaki C, Mobasheri A, Shakibaei M. Synergistic chondroprotective effects of curcumin and resveratrol in human articular chondrocytes: inhibition of IL-1 beta-induced NF-kappaB-mediated inflammation and apoptosis. Arthritis Res Ther 2009;11:R165.

14. Lei M, Liu SQ, Liu YL. Resveratrol protects bone marrow mesenchymal stem cell derived chondrocytes cultured on chitosangelatin scaffolds from the inhibitory effect of interleukin-1beta. Acta Pharmacol Sin 2008;29:1350-6.

15. Liu FC, Hung LF, Wu WL, et al. Chondroprotective effects and mechanisms of resveratrol in advanced glycation end productsstimulated chondrocytes. Arthritis Res Ther 2010;12:R167.

16. Shakibaei M, Csaki C, Nebrich S, et al. Resveratrol suppresses interleukin-1beta-induced inflammatory signaling and apoptosis in human articular chondrocytes: potential for use as a novel nutraceutical for the treatment of osteoarthritis. Biochem Pharmacol 2008;76:1426-39.
17. Tian J, Chen JW, Gao JS, et al. Resveratrol inhibits TNF- $\alpha$-induced IL-1 $\beta$, MMP-3 production in human rheumatoid arthritis fibroblastlike synoviocytes via modulation of PI3kinase/Akt pathway. Rheumatol Int 2013;33:1829-35.

18. Byun HS, Song JK, Kim YR, et al. Caspase-8 has an essential role in resveratrol-induced apoptosis of rheumatoid fibroblast-like synoviocytes. Rheumatology 2008;47:301-8.

19. Ferrero ME, Bertelli $A E$, Fulgenzi $A$, et al. Activity in vitro of resveratrol on granulocyte and monocyte adhesion to endothelium. Am J Clin Nutr 1998:68:1208-14

20. Vorderstrasse BA, Steppan LB, Silverstone AE, et al. Aryl hydrocarbon receptor-deficient mice generate normal immune responses to model antigens and are resistant to TCDD-induced immune suppression. Toxicol Appl Pharmacol 2001;171:157-64.

21. Elmali N, Esenkaya I, Harma A, et al. Effect of resveratrol in experimental osteoarthritis in rabbits. Inflamm Res 2005;54:158-62.

22. Wang J, Gao JS, Chen JW, et al. Effect of resveratrol on cartilage protection and apoptosis inhibition in experimental osteoarthritis of rabbit. Rheumatol Int 2012;32:1541-8.

23. Li W, Cai L, Zhang Y, et al. Intra-articular resveratrol injection prevents osteoarthritis progression in a mouse model by activating SIRT1 and thereby silencing HIF-2 $\alpha$. J Orthop Res 2015;33:1061-70.

24. Amiot MJ, Romier B, Dao TM, et al. Optimization of trans-Resveratrol bioavailability for human therapy. Biochimie 2013;95:1233-8.

25. Schulz KF, Altman DG, Moher D, et al. CONSORT 2010 statement: updated guidelines for reporting parallel group randomised trials. BMJ 2010;340:c332.

26. Cottart $\mathrm{CH}$, Nivet-Antoine V, Laguillier-Morizot $\mathrm{C}$, et al. Resveratrol bioavailability and toxicity in humans. Mol Nutr Food Res 2010;54:7-16.

27. Bellamy N, Kirwan J, Boers M, et al. Recommendations for a core set of outcome measures for future phase III clinical trials in knee, hip, and hand osteoarthritis. Consensus development at OMERACT III. J Rheumatol 1997;24:799-802.

28. McAlindon TE, Driban JB, Henrotin Y, et al. OARSI Clinical Trials Recommendations: Design, conduct, and reporting of clinical trials for knee osteoarthritis. Osteoarthritis Cartilage 2015;23:747-60.

29. Bellamy N, Buchanan WW, Goldsmith $\mathrm{CH}$, et al. Validation study of WOMAC: a health status instrument for measuring clinically important patient relevant outcomes to antirheumatic drug therapy in patients with osteoarthritis of the hip or knee. $J$ Rheumatol 1988;15:1833-40.

30. Pham T, van der Heijde D, Altman RD, et al. OMERACT-OARSI initiative: Osteoarthritis Research Society International set of responder criteria for osteoarthritis clinical trials revisited. Osteoarthritis Cartilage 2004;12:389-99. 